\title{
Words are not enough: nonword repetition as an indicator of arcuate fasciculus integrity during brain tumor resection
}

\author{
Joanna Sierpowska, MSc, ${ }^{1,2}$ Andreu Gabarrós, MD, PhD, ${ }^{3}$ Alejandro Fernandez-Coello, MD, ${ }^{3,4}$ \\ Àngels Camins, MD, ${ }^{5}$ Sara Castañer, MD, ${ }^{5}$ Montserrat Juncadella, $\mathrm{PhD},{ }^{6}$ Joaquín Morís, $\mathrm{PhD},{ }^{1,2}$ and \\ Antoni Rodríguez-Fornells, $\mathrm{PhD}^{1,2,7}$ \\ ${ }^{1}$ Cognition and Brain Plasticity Group, Bellvitge Biomedical Research Institute (IDIBELL), L'Hospitalet de Llobregat; ${ }^{2}$ Department \\ of Basic Psychology, Campus Bellvitge, University of Barcelona, L'Hospitalet de Llobregat; ${ }^{3}$ Hospital Universitari de Bellvitge, \\ Neurosurgery Section, Campus Bellvitge, University of Barcelona, IDIBELL, L'Hospitalet de Llobregat; ${ }^{4} \mathrm{CIBER}$ de Bioingeniería, \\ Biomateriales y Nanomedicina; ${ }^{5}$ Institut de Diagnòstic per la Imatge, Centre Bellvitge, Hospital Universitari de Bellvitge, \\ L'Hospitalet de Llobregat; ${ }^{6}$ Hospital Universitari de Bellvitge, Neurology Section, Campus Bellvitge, University of Barcelona, \\ IDIBELL, L'Hospitalet de Llobregat; and 'Catalan Institution for Research and Advanced Studies, ICREA, Barcelona, Spain
}

OBJECTIVE Subcortical electrical stimulation during brain surgery may allow localization of functionally crucial white matter fibers and thus tailoring of the tumor resection according to its functional limits. The arcuate fasciculus (AF) is a white matter bundle connecting frontal, temporal, and parietal cortical areas that is often disrupted by left brain lesions. It plays a critical role in several cognitive functions related to phonological processing, but current intraoperative monitoring methods do not yet allow mapping of this tract with sufficient precision. In the present study the authors aimed to test a new paradigm for the intraoperative monitoring of the AF.

METHODS In this report, the authors studied 12 patients undergoing awake brain surgery for tumor resection with a related risk of AF damage. To preserve AF integrity and the cognitive processes sustained by this tract in the intraoperative context, the authors used real word repetition (WR) and nonword repetition (NWR) tasks as complements to standard picture naming.

RESULTS Compared with the errors identified by WR or picture naming, the NWR task allowed the detection of subtle errors possibly related to AF alterations. Moreover, only 3 patients demonstrated phonological paraphasias in standard picture naming, and in 2 of these patients the paraphasias co-occurred with the total loss of WR and NWR ability. Before surgery, lesion volume predicted a patient's NWR performance.

CONCLUSIONS The authors suggest that monitoring NWR intraoperatively may complement the standard naming tasks and could permit better preservation of the important language production functions subserved by the AF.

http://thejns.org/doi/abs/10.3171/2016.2.JNS151592

KEY WORDS arcuate fasciculus; electrical stimulation mapping; nonword repetition; dual pathway model for language processing; oncology

$\mathrm{D}$ IRECT electrical stimulation mapping (ESM) is currently a gold standard for defining eloquent language-related cortical brain areas in neurosurgical operations involving tumor and vascular malformation removal. A significant body of literature documents the usefulness of tasks controlling for language performance while direct electrical current is applied to the pa- tient's cortex. Traditionally, language mapping procedures started with simple object-naming tasks. ${ }^{33}$ This simple task allowed one to capture a variety of errors and still is the most commonly used task..$^{12,15,41,42}$ Recently, more developed protocols have come into play allowing for the exploration of more sophisticated facets of language processing such as verb generation, ${ }^{19,33,42}$ spontaneous speech

ABBREVIATIONS AF = arcuate fasciculus; $C A=$ conduction aphasia; $C U S A=$ cavitron ultrasonic surgical aspirator; $D T I=$ diffusion tensor imaging; $E S M=$ electrical stimulation mapping; $\mathrm{MNI}=$ Montreal Neurological Institute; NWR = nonword repetition; ROI = region of interest; Spt = sylvian parietotemporal; WR = word repetition. SUBMITTED July 10, 2015. ACCEPTED February 16, 2016. 
production, ${ }^{12,13}$ comprehension and semantic processing,${ }^{45}$ semantic and phonological fluency, ${ }^{2,54}$ sentence processing, ${ }^{42}$ reading, ${ }^{44}$ and writing. ${ }^{30}$

However, these tasks are mostly designed for ESM procedures at the cortical level, and in most cases the treated lesions do not only affect the cortex but also infiltrate the deep white matter, disturbing crucial subcortical connectivity. For this reason and to prevent potential postoperative impairments, it is important to evaluate the function of the most important white matter fiber bundles adjacent to resected tumor during electrical subcortical stimulation. In this context the routine use of neuropsychological monitoring at the subcortical white matter level has been proposed, but until presently it has been mainly described in unique case studies of particular patients. ${ }^{12,14,15,29}$ In the present study, we focus on monitoring arcuate fasciculus (AF) function, considering its crucial role in the correct transfer of information and conversion of sounds (phonology) into articulatory output patterns (language production).

The AF is a white matter bundle connecting posterotemporal, inferoparietal, and prefrontal (mostly premotor and inferofrontal language-related) regions. ${ }^{6,7}$ Broadly understood, the AF plays an important role in language learning,,$^{24}$ singing and musical training,${ }^{18}$ speech production, ${ }^{26}$ repetition, ${ }^{36}$ and phonological processing. ${ }^{47}$ According to the classic models of language organization, this tract connecting the Broca and Wernicke areas ("long segment of the AF" in the current nomenclature) has been associated with language repetition, and its disruption is related to the symptoms associated with conduction aphasia. Considering the proposed dual stream organization of language processing, the AF forms a crucial part of the so-called dorsal language pathway, which is related to the mapping of sounds into their articulatory representations. ${ }^{20,40,46,57}$

Bearing in mind the importance of the AF in preserving language competence postoperatively, we aimed to evaluate for the first time to what extent a nonword repetition (NWR) task might allow more reliable mapping of functions related to the AF, both preoperatively and intraoperatively. The importance of the repetition of real words in intraoperative monitoring was highlighted by MoritzGasser and Duffau in 2013. ${ }^{29}$ However, the repetition of words might be accomplished using redundant and preserved lexical retrieval pathways that are not directly associated with the recruitment of the dorsal language pathway mentioned above. This is not the case for NWR, as no lexical trace exists in memory, and therefore the auditory nonword representation must be transformed directly into an articulatory representation without the mediation of existing vocabulary. In the present study, nonwords were created using letter substitutions in real words and following Spanish phonotactic constraints, resulting in nonwords that were easy to articulate. Nonwords are considered useful when exploring language mechanisms in patients presenting with syndromes such as poststroke aphasia, ${ }^{3}$ primary language impairment, ${ }^{28}$ specific language impairment, ${ }^{9,17}$ hearing impairments, ${ }^{21}$ stuttering, ${ }^{4}$ Alzheimer's disease, ${ }^{31}$ or semantic dementia. ${ }^{22}$

We tested the role and reliability of an NWR task as an indicator of AF integrity in comparison with standard word repetition (WR) and picture-naming tasks. We evaluated its usefulness in a systematic case study of 12 patients harboring intrinsic brain lesions and undergoing awake brain surgery. Task performance was combined with the precise location of the AF using neuronavigation.

\section{Methods Sample}

During the period between 2012 and 2014, 12 patients (9 males) in the Neurology Ward of the Hospital Universitari de Bellvitge, Barcelona, Spain, were included in this study. Criteria for inclusion were an age between 18 and 65 years, a minimum score of $60 \%$ in the homemade picture-naming task, and surgical exposure involving the cortical terminations and/or white matter connection of the left AF. Four patients harbored low-grade tumors; 6 patients, high-grade tumors; 1 patient, metastasis of the parotid gland; and 1 patient, a cavernous angioma (Table 1). Patient ages ranged from 18 to 64 years (mean \pm SD, $44 \pm 15.7$ years), and their education ranged from 6 to 14 years (mean $10.42 \pm 2.54$ years). Ten patients were righthanded and 2 were left-handed according to the standard Edinburgh Inventory (right-handedness was not an inclusion criterion since the dominance for language was further confirmed by presurgical functional MRI assessment). Each patient signed informed consent to participate in this study, and the ethics committee of the hospital approved the protocol.

\section{Brain Imaging Data}

Anatomical (T1-weighted and diffusion tensor) images were collected at the Hospital Universitari de Bellvitge before surgery. The MRI equipment consisted of a Philips Intera 1.5-T system with a maximum field gradient strength of $76 \mathrm{mT} / \mathrm{m}$. High-resolution T1-weighted images were acquired using the following parameters: slice thickness $1.1 \mathrm{~mm}$, number of slices 150, TR $25 \mathrm{msec}$, TE 4.6 msec, flip angle $30^{\circ}$, matrix $320 \times 320$, FOV $240 \mathrm{~mm}$, and voxel size $0.75 \times 0.75 \times 1.1 \mathrm{~mm}$. Diffusion tensor imaging (DTI) data were obtained using a single-shot echo planar imaging sequence. Diffusion gradients were applied along 16 directions using a b-value of 0 and $800 \mathrm{sec} / \mathrm{mm}^{2}$. The DTI sequences were acquired in the axial plane with 60 contiguous sections, a 2-mm section thickness (voxel size $1.63 \times 1.63 \times 2 \mathrm{~mm}$ ), no intersection gap, TR $15,600 \mathrm{msec}$, TE $79 \mathrm{msec}$, FOV $170 \times 234 \mathrm{~mm}^{2}$, and matrix $84 \times 117$. These sequences allowed the assessment of presurgical damage and supported intraoperative neuronavigation.

We calculated lesion volume by creating a binary mask outlining the lesion's precise location in the MRIcron software package ${ }^{43}$ in the native space of the 3D T1-weighted images (Table 1) and by multiplying the number of voxels composing the lesion by the voxel size. Further, we normalized the lesion mask to the Montreal Neurological Institute (MNI) space and overlapped them for visualization purposes (Fig. 1). To verify in which patients the lesion may have involved the AF, we followed the methodology described by Thiebaut de Schotten et al., ${ }^{52}$ using the Tractotron software. For this purpose, we manually delineated 
TABLE 1. Summary of patient and brain lesion characteristics and AF fiber alteration patterns

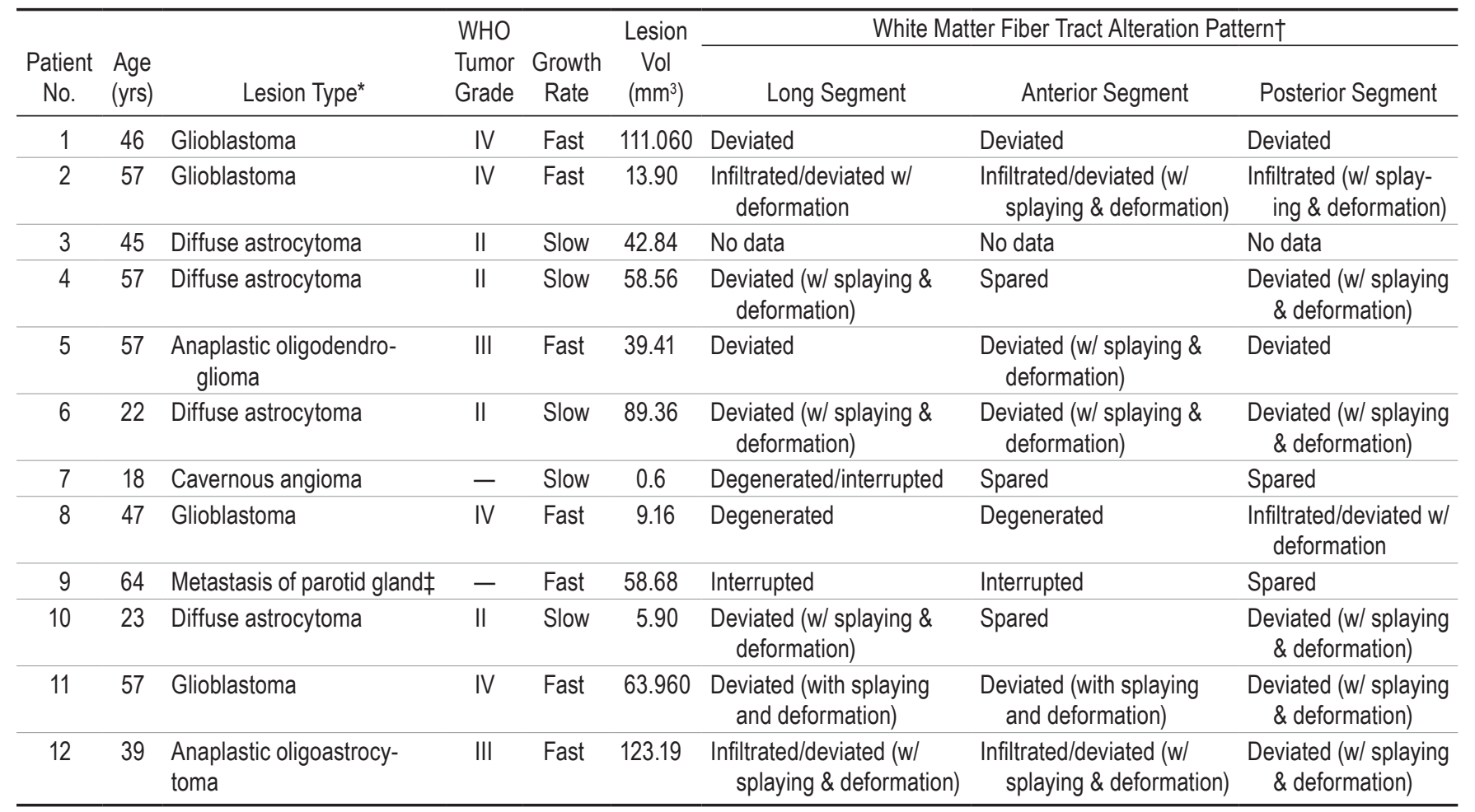

\footnotetext{
* Tumor type established according to the 2007 WHO classification of tumors of the central nervous system (Louis et al., 2007).

$\dagger$ Patterns of white matter alterations defined according to the study by Lazar et al., 2006.

$\ddagger$ Metastasis of the parotid gland is not graded according to the WHO scale, but being malignant can also be considered as rapidly growing (Olsen and Lewis, 2001).
}

the lesions in the native space of T1-weighted images of the patient brains using MRIcron software and then normalized them to the MNI coordinates. Further, the normalized lesion maps were compared with an a priori MNI atlas depicting the distribution of white matter tracts in normal brain..$^{8,51,52}$ This DTI atlas provides a probability for each voxel to belong to a specific tract in the MNI space and can be used for a voxel by voxel comparison between the brain harboring a lesion and the template of white matter tract distribution. The tract is considered to be disconnected if the overlap between a lesion and the atlas-derived tract coordinates surpasses $50 \%$ (chance level)..$^{52}$

\section{Diffusion Tensor Imaging Dissections}

Virtual in vivo DTI dissections of the 3 segments of the AF were performed in the native space using the color fiber-orientation fractional anisotropy maps implemented in the TrackVis software. ${ }^{55}$ Tracts were delineated using the 2 regions-of-interest (ROIs) approach and according to a previously reported methodology. ${ }^{6,7,53}$ The 2 ROIs approach permitted visualization of the fibers that connect 2 specific brain regions. In this study, the ROIs were delineated according to each patient's individual brain anatomy, and their size was limited to these specific anatomical landmarks; however, in patients whose normal tract trajectory was displaced by tumor, the ROIs were enlarged or moved following the color-coded map guidance (Supplemental Figure A). Moreover, while connecting the 2 ROIs, the fibers were not constrained to end within the ROI; they were allowed to freely project until they reached the cortex. The first ROI was delineated in the coronal plane, anteriorly to the coronal fissure. The bundle of fibers appearing in green in the color-coded map was delineated using a circular hand-drawn 2D ROI just before the fibers turned to meet the cortex (frontal ROI). A similar, 2D hand-drawn ROI was then placed in the axial plane encompassing the fibers descending to the posterior temporal lobe through the posterior portion of the temporal stem (blue fibers in the color-coded map; temporal ROI). Finally, the parietal ROI was placed in the sagittal plane around the angular and supramarginal gyri. This last ROI was also 2D and limited only to these particular gyri.

Each segment of the AF was defined by the combination of 2 of these 3 ROIs. The fibers passing through the frontal and temporal ROIs were classified as the long segment of the AF (red), the streamlines passing through the temporal and parietal ROIs constituted the posterior segment of the $\mathrm{AF}$ (yellow), and the streamlines passing through the frontal and parietal ROIs formed the anterior segment of the AF (green; Fig. 1 and Supplemental Figure A).

\section{Electrical Stimulation Mapping}

In the cortical ESM procedure, we followed the guidelines proposed by Ojemann et al. ${ }^{32}$ and described in our previous publications. ${ }^{48,49}$ At the subcortical level, tumor resection and stimulation of the adjacent structures were achieved using a cavitron ultrasonic surgical aspirator 

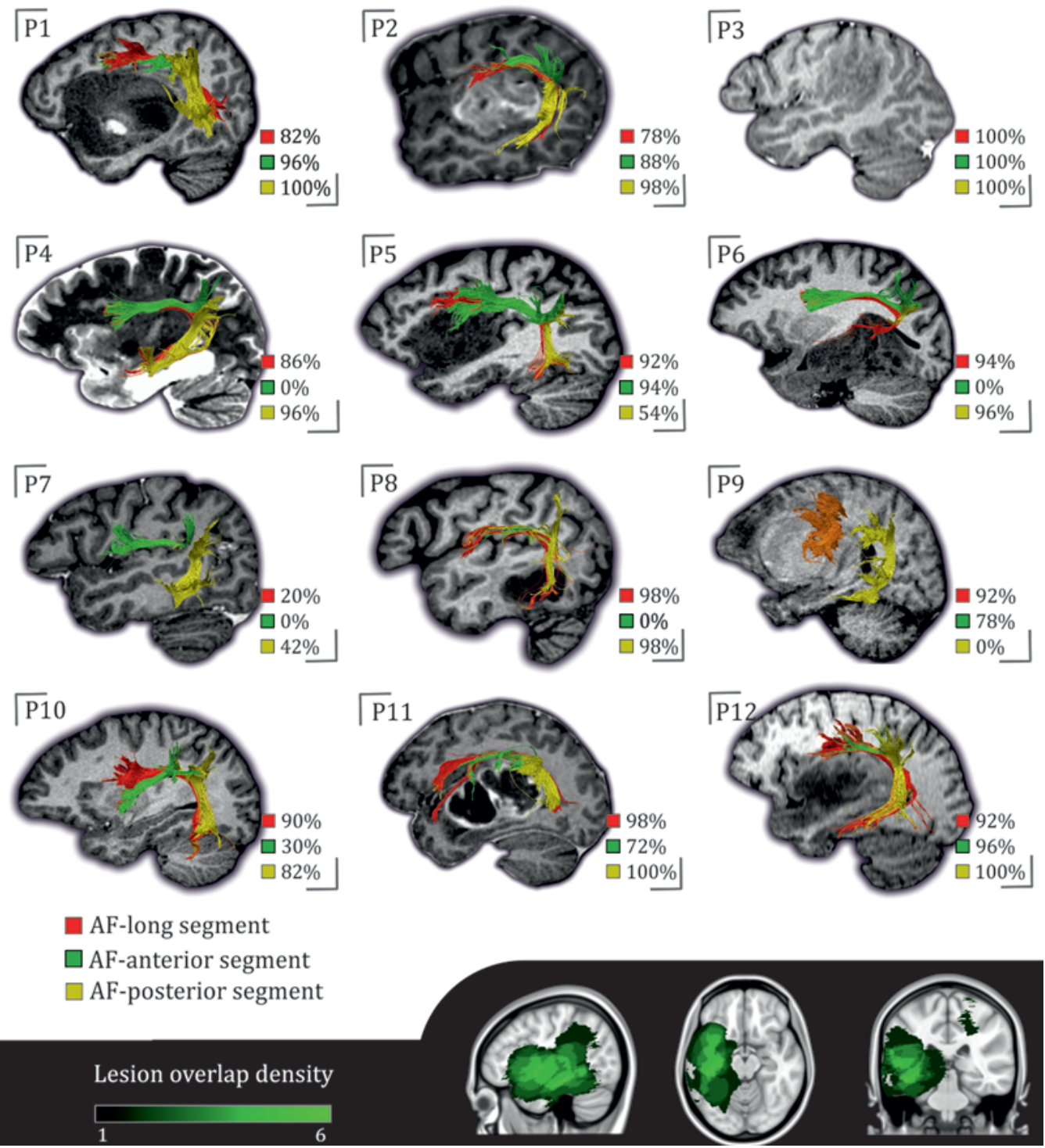

FIG. 1. Presurgical visualization (upper) of lesions on T1-weighted MRI (T2-weighted MRI for Patient 4 [P4]) and the 3 segments of the AF on in vivo tractography (DTI raw data for P3 unavailable). The key to right of each image indicates the probability of damage to each segment of the AF as calculated using the Tractotron software. Maps of the lesion overlap density (lower) of the entire sample of 12 patients. Figure is available in color online only.

(CUSA). The CUSA is composed of an ultrasound generator, a sucker, and an irrigator that together form an ultrasonic dissector/aspirator selective for tissue with high water and low collagen content and serves as a fundamental tool for the surgical removal of tumors. ${ }^{5}$ Moreover, it has also been seen to cause stimulation of tissue adjacent to tumor comparable to the stimulation induced by the Ojemann cortical stimulator in ESM. Carrabba and colleagues reported that the CUSA provoked a transient inhibition of motor function during brain tumor removal in a 44-yearold patient harboring recurrent oligoastrocytoma. ${ }^{5}$ In this patient, continuous multichannel electromyography recorded motor responses each time the CUSA was turned on in the vicinity of the motor tracts; if the device was turned off, the response faded. The exact neurophysiological mechanism of this interference remains unclear, but the transient inhibition of axonal conduction has been proposed as a possible explanation. The important advantage of CUSA stimulation is that neuropsychological assessment can be performed simultaneously to tumor resection; thus, there is no need to prolong the real time of the operation. The authors reported that their case study on motor function was designed in an attempt to explain the occurrence of language errors during CUSA-aided tumor removal simultaneous to speech assessment. The present study follows this rationale in trying to explore how CUSA stimulation of language tracts adjacent to tumor may be controlled through the suitable selection of intraoperative tasks. It is important to disentangle differences in the performance of language tasks depending on whether the CUSA induced a transient inhibition of the tissue adjacent to tumor or, when the damage is irreversible, if the tract 
was infiltrated by tumor. We addressed this issue in Table 1 by indicating the tract alteration patterns assessed in the presurgical in vivo DTI dissections. Note that spared tracts or preoperative deviation (without infiltration) patterns in at least 1 of the $3 \mathrm{AF}$ segments were observed in 9 of 12 patients (1 DTI data set was missing). The degeneration, infiltration, or interruption patterns were observed in 5 of 11 patients (Patients 2,7-9, 12). One of these 5 patients harbored a very small cavernous angioma (Patient 7) at a considerable distance from the lesion, so it is possible that the absence of the long segment of the AF is attributable to another etiology. Importantly, the remaining 4 patients presenting with the degeneration, infiltration, or interruption patterns were harboring the fast-growing lesions.

During the operation, both anatomical and functional monitoring were performed. Surgeons controlled the precision of the removal by using a neuronavigation system (BrainLab), and neuropsychologists controlled language function using appropriate language processing tasks. Neuronavigation allowed monitoring of the location using both anatomical T1-weighted images for the gray matter sulci and in vivo DTI reconstructions for white matter subcortical fasciculi. Each time the level of the AF vicinity was reached during the operation, the repetition tasks were administered to the patient simultaneously to the CUSA action.

\section{Neuropsychological Assessment}

Standard neuropsychological assessment was performed in the Neurology Ward of Hospital Universitari de Bellvitge before and after surgery. In this protocol we included measures of handedness (Edinburgh Inventory ${ }^{34}$ ), verbal comprehension (Token Test ${ }^{11}$ ), semantic (animals) and phonological (letter p) verbal fluency, ${ }^{16}$ attention and working memory (Digit Span from the Wechsler Adult Intelligence Scale ${ }^{56}$ ), and naming (Boston Naming Task ${ }^{16}$ ). Moreover, to screen patients for the surgical procedures, we designed a simplified version of the picture-naming task, which was composed of 60 black and white drawings selected from a standard stimuli database..$^{50}$

\section{Tasks: WR and NWR}

The WR and NWR tasks were both designed to control phonological processing. For the WR task, 40 Spanish words were selected from the LEXESP database..$^{10}$ Twenty-eight of these words were bisyllabic and 12 were trisyllabic; all of them were selected to diversify their frequency (mean estimated lexical frequency $19.25 \pm 19.07 /$ million words in the LEXESP corpus). The nonwords were derived from the aforementioned set of 40 words, respecting Spanish phonotactical rules and using letter substitutions; for example, from the real word "camisa" (shirt) we created a nonword "lavica." We screened the patients for their repetition abilities before surgery by using the complete set of 40 words and 40 nonwords. During the repetition task, a female experimenter read the items aloud, and the same task was performed before, during, and after surgery. The word or nonword was considered correctly repeated if each single phoneme was reproduced in exactly the same manner as the experimenter within 3 seconds of the stimulus presentation. Self-correction was not scored, and a single repetition of stimuli was allowed on the patient's request (and when an uncontrolled external noise occurred in the consulting or operating room). An audio recording of task performance was obtained. After the presurgical assessment, all items incorrectly repeated were excluded from the intraoperative set, so the errors observed during surgery were most likely provoked by the intraoperative manipulations and not by other factors. To obtain data on WR and NWR task performance from participants free from neurological conditions, we performed both tasks in a group of 30 healthy native Spanish speakers (mean age $29.4 \pm 6.22$ years, 20 females).

\section{Statistical Analysis}

We tested potential differences in each patient's performance in the WR and NWR tasks at 3 time points: before, during, and after surgery. When testing these withinparticipant measures, the nonparametric Wilcoxon test for paired samples was used. To explore the relation between lesion volume and scores on a particular repetition task we used the Pearson correlation coefficient. All these statistical tests were 2-tailed with a critical $\mathrm{p}$ threshold of 0.05 . To test whether the relationship between WR and NWR performance in healthy subjects significantly differed from that in patients, we ran a mixed repeated-measures ANOVA with a within-subject factor (that is, stimulus type: WR and NWR) and a between-subjects factor (that is, group: patients and healthy subjects). This interaction was further analyzed with 2 post hoc t-tests corrected for unequal variances. Statistical analyses were performed using SPSS PASW Statistics 18 (PASW Statistics for Windows, SPSS Inc.).

\section{Results \\ Lesion Sites}

Visual inspection of the presurgical neuroimaging data confirmed that patient's lesions were located in the areas including the AF, its cortical terminations, or within the adjacent white matter (Fig. 1 and Table 1). In addition, we used Tractotron software to estimate the percentage of probability of AF damage (based on the distribution of voxels defining the lesion within the MNI space ${ }^{52}$ ). Overall in our sample, the probability of damage to the long segment of the AF was $85.17 \%, 54.5 \%$ to the anterior segment, and $80.5 \%$ to the posterior segment (Table 2). While considering each patient individually, we observed that the probability of the disconnection of at least 2 segments surpassed 50\% (chance level) in all patients except Patient 7. However, bearing in mind that the virtual in vivo dissections in this particular patient revealed that the left long segment of the AF was missing (and thus the more important the mapping of the remaining 2 segments), we decided to include her in the final sample. In a considerable number of patients (Patients 1, 2, 4, 5, 10, and 12), the results from Tractotron indicated a probability of damage near $100 \%$, but indeed the tract was preserved. On these occasions, it is important to account for the fact that the Tractotron software permits one to calculate the probability of tract disruption assuming that the tracts are placed 
TABLE 2. Percentage of the probability of left AF damage*

\begin{tabular}{ccccc}
\hline Parameter & $\begin{array}{c}\text { Long } \\
\text { Segment }\end{array}$ & $\begin{array}{c}\text { Anterior } \\
\text { Segment }\end{array}$ & $\begin{array}{c}\text { Posterior } \\
\text { Segment }\end{array}$ & $\begin{array}{c}\text { Mean for } \\
\text { Patient }\end{array}$ \\
\hline Patient No. & & & & \\
\hline 1 & 82 & 96 & 100 & 92.7 \\
\hline 2 & 78 & 88 & 98 & 88 \\
\hline 3 & 100 & 100 & 100 & 100 \\
\hline 4 & 86 & 0 & 96 & 60.7 \\
\hline 5 & 92 & 94 & 54 & 80 \\
\hline 6 & 94 & 0 & 96 & 63.3 \\
\hline 7 & 20 & 0 & 42 & 20.7 \\
\hline 8 & 98 & 0 & 98 & 65.3 \\
\hline 9 & 92 & 78 & 0 & 56.7 \\
\hline 10 & 90 & 30 & 82 & 67.3 \\
\hline 11 & 98 & 72 & 100 & 90 \\
\hline 12 & 92 & 96 & 100 & 96 \\
\hline Mean for tract $\dagger$ & $85.2 \pm 21.53$ & $54.5 \pm 44.24$ & $80.5 \pm 32.02$ & \\
\hline
\end{tabular}

* Calculated using Tractotron and according to the presurgical lesion masks delineated on anatomical T1-weighted MR images. The tract may be considered as disconnected if the probability of its damage surpasses $50 \%$ (chance level; see Results for the reason for including Patient 7 in the final sample). † Mean \pm standard deviation.

within coordinates based on the atlas of the normal sample. In a brain harboring a tumor, the tracts are often displaced (Table 1), which implies that, according to Tractotron, the tracts can be considered as disrupted simply because they are not situated in their correct position.

\section{Intraoperative WR and NWR Performance}

As expected, during surgery patients committed significantly more errors on the NWR task than the WR task (mean percentage of errors: WR $9.04 \pm 17.14$ vs NWR 29 $\pm 8.9, \mathrm{Z}=2.67, \mathrm{p}=0.008$; Fig. 2). Note that the occurrence of intraoperative phonological errors on the standard picture-naming task was virtually absent in all but 3 patients (overall mean percentage of errors $0.49 \pm 0.75$ ). Interestingly, 2 (Patients 11 and 12) of the 3 aforementioned patients were unable to repeat any of the target words and nonwords during tumor resection; thus, these tasks were not used intraoperatively in these 2 cases. Nevertheless, to continue intraoperative monitoring, the neuropsychologist used the standard picture-naming task, in which the proportion of phonological errors (paraphasias) was very high for both patients (Patient 11: $18.52 \%$ and Patient 12: 17.22\%; these scores are indicated by a black dashed circle in Fig. 2).

\section{Presurgical and Postsurgical Neuropsychological Assessment}

As shown in Table 3, presurgical performance on the NWR task was more impaired than on the WR (presurgery: $\mathrm{Z}=2.94, \mathrm{p}=0.003$ ). Importantly, we observed that the lesion volume predicted the level of preoperative impairment on the NWR task $(r=0.58, p<0.05$; Fig. 3$)$ but not on the WR task $(r=0.49, p>0.05)$. After surgery the same WR and NWR tasks were performed in patients to

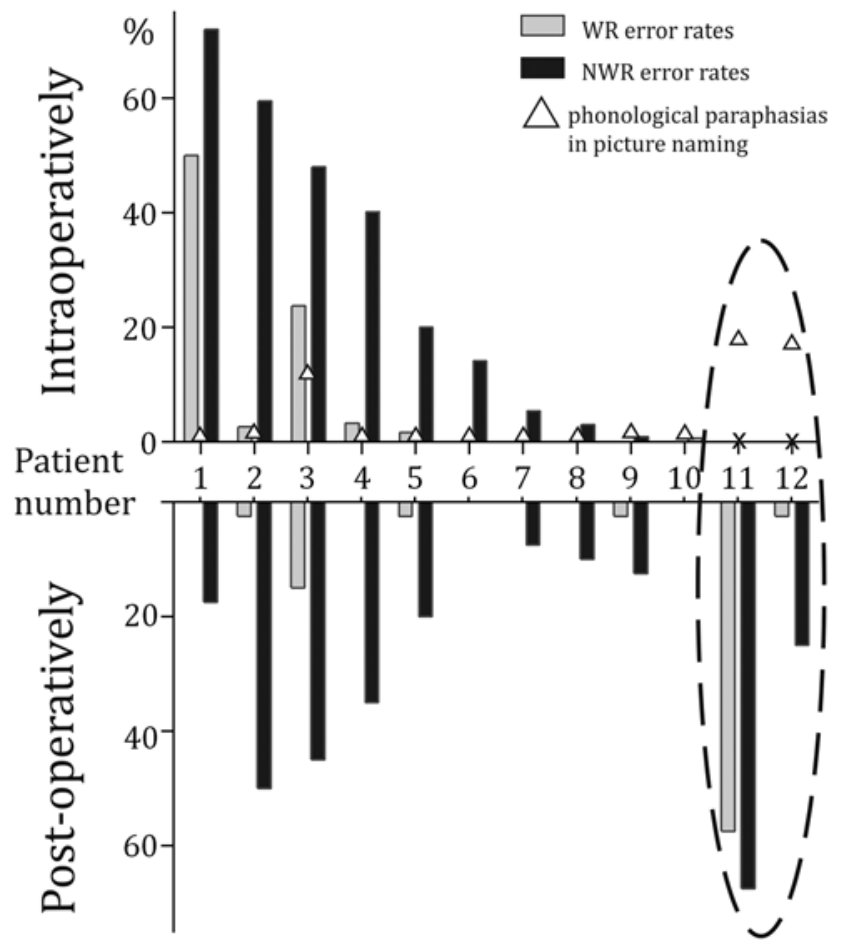

FIG. 2. Intraoperative (upper) and postoperative (lower) error rates on the WR, NWR, and picture-naming tasks (phonological paraphasias). Note that Patients 11 and 12 (black dashed circle) were unable to perform the WR and NWR tasks intraoperatively, while at the same time presenting the highest level of phonological paraphasias in the picture-naming task and a severe postsurgical impairment. Note also that a great number of patients did not produce errors in the WR task, as opposed to the NWR task, which also showed greater intersubject variability.

control for possible postoperative changes and revealed that at the group level there was no significant deterioration in any of the language repetition measures (WR and NWR). However, after surgery the patients again committed more errors on the NWR task than on the WR task $(\mathrm{Z}=2.81, \mathrm{p}=0.005)$. Standard neuropsychological assessment scores for the patient group both before and after surgery are presented in Table 3. Importantly, a comparison between these standard neuropsychological measures preand postoperatively did not reveal statistically significant differences $(Z<1.49, p>0.14)$. The performance of repetition tasks by a group of 30 healthy subjects was flawless on the WR task (100\% accuracy) and virtually perfect on the NWR task (98.6\% accuracy); compare these results to patient group performance before surgery: $98.5 \%$ accuracy on WR and $79.8 \%$ accuracy on NWR. A comparison between patient group and healthy group performance using mixed ANOVA revealed that there was a main effect of stimulus type (WR vs NWR, F[1,40] = 72.82, $\mathrm{p}<0.001$ ) and group (patients vs healthy subjects, $F[1,40]=46.86, \mathrm{p}$ $<0.001)$. There was also a significant interaction between stimulus type and group: $\mathrm{F}(1,40)=53.797, \mathrm{p}<0.001$.

An additional analysis showed that the difference between WR and NWR error rates was significantly higher in the patient group than in healthy controls $(\mathrm{t}[11.1]=4.61$, $\mathrm{p}<0.001)$. 


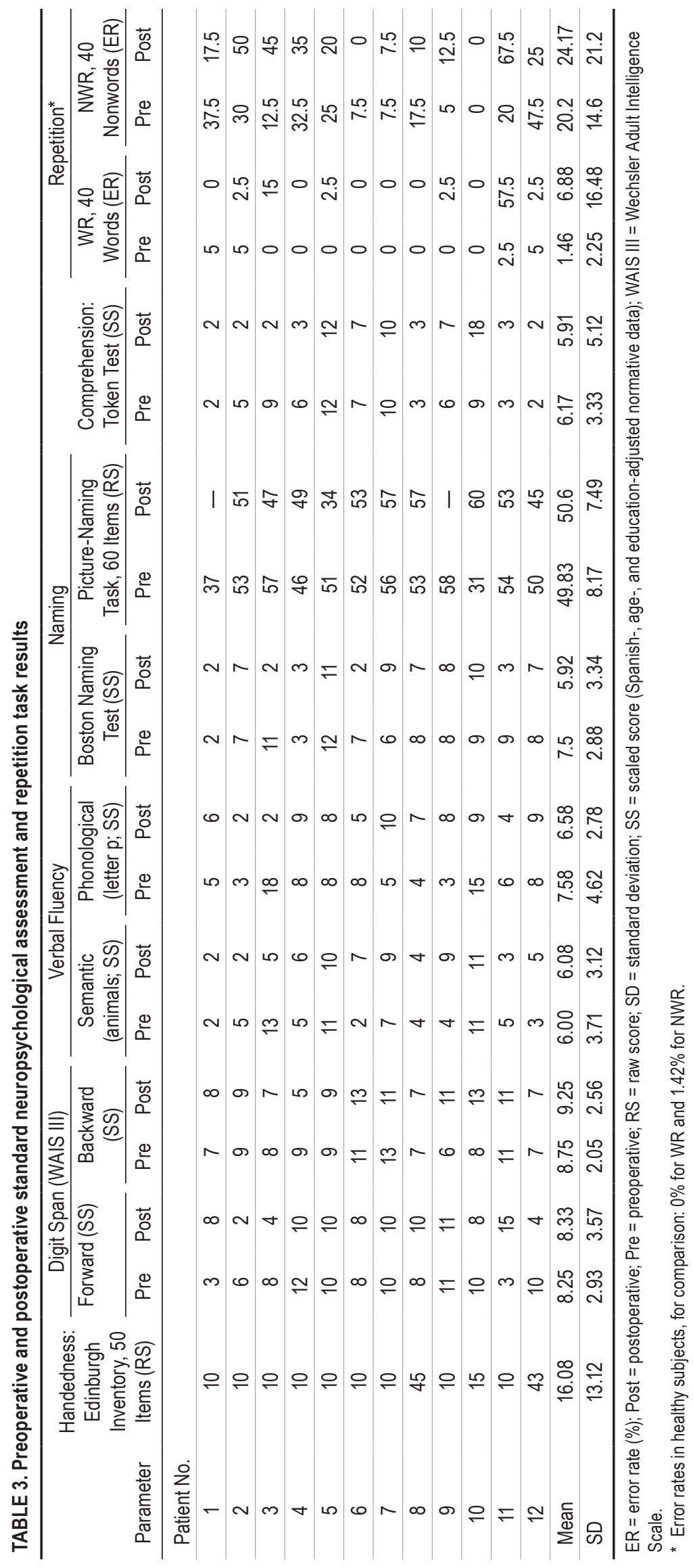




\section{Discussion}

Here we report on a systematic case series of 12 patients whose surgical intervention was related to the risk of AF integrity disruption. Within this patient group, we detected significantly more errors using the NWR task than the WR task (pre-, intra-, and postoperatively). These results may indicate a greater sensitivity of the NWR task to a possible white matter disruption during surgeries involving the perisylvian language areas and are in line with the observations by Moritz-Gasser and Duffau, ${ }^{29}$ who stated that the repetition of real words was inefficient for effective AF monitoring.

Nonwords bearing no semantic content make the repetition slightly more difficult even for healthy subjects. However, we observed that the difference between WR and NWR is significantly more pronounced in patients harboring a brain lesion in the left perisylvian languagerelated areas than in healthy subjects.

Importantly, lesion volume (note that the lesions were located in the AF vicinity; Fig. 1) in our patients significantly predicted the error rate on the presurgical NWR task. These results converge with those of a previous study in which the impairment of speech production was associated with a left AF lesion load (extent of overlap between the AF and the patient's lesion ${ }^{26}$ ) and indicate that the bigger the lesion, the more prominent the impairment of the functional network.

Hitherto, the loss of AF integrity during brain surgery was mainly detected using the conventional picture-naming task and was related to the appearance of phonological paraphasias during low-grade tumor resections. ${ }^{14}$ Although this evidence converges with our results, it is important to note that in our patients, the naming task allowed the detection of phonological paraphasias intraoperatively in only 3 severe cases ( 2 of which co-occurred with the total loss of repetition capacity, precluding the intraoperative assessment of repetition in Patients 11 and 12). Therefore, these results suggest that the widely used intraoperative picture-naming task should be complemented with other language tasks that more specifically target language processes supported by specific white matter pathways, as was the case in the present study of the AF and NWR. Importantly, it was previously observed that AF injury is related not only to repetition impairment, but also to difficulties in other language domains. For example, radiationinduced tissue necrosis damage to the $\mathrm{AF}$ acquired after the age of 5 years led to poor performance not only in NWR, but also in verbal working memory and reading. ${ }^{39}$ Additionally, Shinoura et al. ${ }^{47}$ reported that in 3 patients harboring a lesion affecting the AF, "almost all language functions closely related to phonology were damaged." Finally, a recent lesion study in 8 patients having poststroke damage within the vicinity of this tract revealed selective auditory repetition difficulties across the entire sample. ${ }^{36}$ Classically, AF disruption has been associated with repetition impairment observed in conduction aphasia (CA), and interestingly patient performance in repetition may differ depending on whether they are confronted with words or nonwords. For example, Berthier and colleagues ${ }^{3}$ reported on a stroke patient with CA who repeated words normally but could not repeat nonwords. Beforehand, McCarthy and

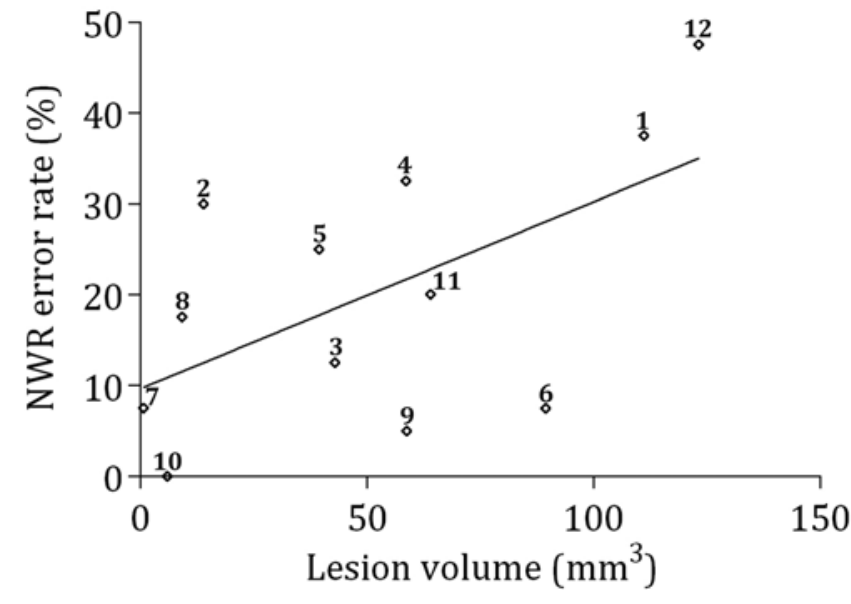

FIG. 3. The relationship between lesion volume and preoperative NWR error rate score. Numbers within the graph represent patient numbers.

Warrington ${ }^{27}$ observed that 2 patients with long segment $\mathrm{AF}$ damage and diagnosed CA repeated better if the task required active semantic processing, whereas their passive repetition was hampered. To explain these differences the authors proposed that the repetition of material differing in its semantic content might rely on distinct neural mechanisms. In this sense, the dorsal pathway (and thus the AF, in forming part of it) would be in charge of phonological processing, and the ventral pathway would be related to the processing of semantic information (dual pathway model for language processing ${ }^{20,40,46,57}$ ). Along the same line, we believe that, to some extent, our WR and NWR tasks may be useful when testing this model intraoperatively, and we suppose that the quantitative differences in scores between WR and NWR may be in some way related to the dual pathway organization for language processing.

Interestingly, and challenging the classically accepted association between repetition and AF white matter, some authors have reported that the symptoms representative of CA may be elicited by the electrical stimulation of the sylvian parietotemporal (Spt) region, already at the cortex level. ${ }^{1,37,38}$ However, in contrast to these electrical stimulation results, a study by Parker Jones and colleagues ${ }^{36}$ revealed that in 8 patients presenting with selective difficulties in repetition, the Spt region was damaged in only one of them, whereas there were lesions affecting the vicinity of the $\mathrm{AF}$ in the entire sample.

From a clinical point of view, our study reveals that WR, NWR, and picture-naming tasks differ significantly in their ability to detect language production errors during intraoperative stimulation of the AF vicinity, with the NWR task being the most sensitive to possible AF damage. Moreover, our clinical observations indicated that this error detection capacity is not only gradual, but is also discontinuous in the sense that in certain cases a patient could lose the ability to correctly repeat nonwords before being unable to repeat real words (most probably using compensatory lexical-semantic brain pathways). Moreover, in 2 patients, we observed a large number of phonological paraphasias during picture naming once these patients had totally lost their ability to repeat. Bearing these observations in mind, we believe that it may be important 
to alternate the use of picture-naming tasks with WR and NWR tasks to localize functional AF sites. Increasing the error detection rates may enable adequate mapping of the critical AF parts and limit further resection by the neurosurgeon. Intraoperative monitoring of the AF seems to be crucial as this white matter tract has been associated with several cognitive functions such as language learning, musical training, phonological working memory, reading, WR, and NWR.

One could argue that the patients' lesions were not limited solely to the AF; thus, the present results could be related to a more complex pattern of disruptions. However, we need to account for the fact that in a clinical practice it is extremely unusual to meet patients with lesions compromising single structures, and we aim to design a neuropsychological assessment protocol adjusted to real clinical demands. Since the aforementioned limitation is especially valid in patients harboring sizable lesions, we added lesion volume as a regressor in our analysis, revealing a strong relationship with preoperative NWR score.

While defining the structures involved in the observed pattern of neuropsychological impairments, we relied on preoperative T1-weighted MRI and DTI, intraoperative stimulation via the CUSA, and intraoperative neuronavigation. Each of these techniques presents its own set of limitations.

First, in relation to preoperative brain imaging, it is important to mention that in brain tumors we need to account for the possible signal distortions as well as the reliability of the ROI placements in the virtual in vivo dissections. To respond to these limitations at least partially, we performed a meticulous visual inspection of fiber alteration patterns, always accounting for the tumor speed of growth, tumor size, and tumor type (Table 1). The virtual in vivo DTI dissections were all performed by the same experimenter and according to a very strict, previously reported set of constraints.

Intraoperatively, involvement of the AF in surgical manipulations (both resection and adjacent stimulation) was controlled by the conjunction of the subcortical CUSA action and neuronavigation simultaneous to a tailored neuropsychological assessment. Given previous evidence about the effects of CUSA action on tumoral and peritumoral tissues, ${ }^{5}$ we assumed that when the tumor resection approached the vicinity of the AF, there would be a need for neuropsychological monitoring. However, if one accounts for the unclear mechanism of CUSA action in language assessment procedures and intraoperative brain shifting, our approach may still lack sufficient precision. To strengthen the evidence for a relationship between AF integrity and NWR disruption, a new protocol for more precise anatomical guidance must be designed. Moreover, the present results should be compared with the performance of patients whose lesions spare AF integrity (for example, right hemisphere lesions). We believe that in this manner NWR may substantially add to our understanding of the neural basis of models of auditory WR and NWR (the paths mediating the transformation of auditory input into articulatory output patterns) and thus, in the future, diminishing the postsurgical impairment in patients harboring tumors in perisylvian language-related regions.

\section{Conclusions}

In this study, 12 patients undergoing language monitoring during awake brain surgery in the left hemisphere (AF vicinity) committed significantly more errors in the NWR task than the WR or picture-naming task before, during, and after surgery, and the preoperative errors on WR and NWR tasks were significantly more pronounced in patients than in healthy subjects.

We propose the NWR task complementing the WR and picture-naming tasks as an easy and reliable screening procedure that may allow one to monitor language processing more carefully during awake brain surgery. However, to strengthen evidence of the relationship between AF integrity and NWR disruption, new protocols must be designed and this question must be addressed in future studies.

\section{Acknowledgments}

We thank Claudia Peñaloza for her constructive remarks on Spanish nonword formation. This project was supported by a Catalan Government award (A.R.F.) and a Spanish Government predoctoral grant (J.S.).

\section{References}

1. Anderson JM, Gilmore R, Roper S, Crosson B, Bauer RM, Nadeau S, et al: Conduction aphasia and the arcuate fasciculus: A reexamination of the Wernicke-Geschwind model. Brain Lang 70:1-12, 1999

2. Bello L, Gallucci M, Fava M, Carrabba G, Giussani C, Acerbi F, et al: Intraoperative subcortical language tract mapping guides surgical removal of gliomas involving speech areas. Neurosurgery 60:67-82, 2007

3. Berthier ML, Froudist Walsh S, Dávila G, Nabrozidis A, Juárez Y Ruiz de Mier R, Gutiérrez A, et al: Dissociated repetition deficits in aphasia can reflect flexible interactions between left dorsal and ventral streams and gender-dimorphic architecture of the right dorsal stream. Front Hum Neurosci 7:873, 2013

4. Byrd CT, McGill M, Usler E: Nonword repetition and phoneme elision in adults who do and do not stutter: vocal versus nonvocal performance differences. J Fluency Disord 44:1731,2015

5. Carrabba G, Mandonnet E, Fava E, Capelle L, Gaini SM, Duffau H, et al: Transient inhibition of motor function induced by the Cavitron ultrasonic surgical aspirator during brain mapping. Neurosurgery 63:E178-E179, 2008

6. Catani M, Allin MPG, Husain M, Pugliese L, Mesulam MM, Murray RM, et al: Symmetries in human brain language pathways correlate with verbal recall. Proc Natl Acad Sci U S A 104:17163-17168, 2007

7. Catani M, Jones DK, ffytche DH: Perisylvian language networks of the human brain. Ann Neurol 57:8-16, 2005

8. Catani M, Thiebaut de Schotten M: Atlas of Human Brain Connections. Oxford: Oxford University Press, 2012

9. Coady JA, Evans JL: Uses and interpretations of non-word repetition tasks in children with and without specific language impairments (SLI). Int J Lang Commun Disord 43:1-40, 2008

10. Davis CJ, Perea M: BuscaPalabras: a program for deriving orthographic and phonological neighborhood statistics and other psycholinguistic indices in Spanish. Behav Res Methods 37:665-671, 2005

11. De Renzi E, Faglioni P: Normative data and screening power of a shortened version of the Token Test. Cortex 14:41-49, 1978

12. De Witte E, Satoer D, Colle H, Robert E, Visch-Brink E, 
Mariën P: Subcortical language and non-language mapping in awake brain surgery: the use of multimodal tests. Acta Neurochir (Wien) 157:577-588, 2015

13. Duffau H, Capelle L, Denvil D, Sichez N, Gatignol P, Taillandier L, et al: Usefulness of intraoperative electrical subcortical mapping during surgery for low-grade gliomas located within eloquent brain regions: functional results in a consecutive series of 103 patients. J Neurosurg 98:764-778, 2003

14. Duffau H, Moritz-Gasser S, Mandonnet E: A re-examination of neural basis of language processing: proposal of a dynamic hodotopical model from data provided by brain stimulation mapping during picture naming. Brain Lang 131:1-10, 2014

15. Fernández Coello A, Moritz-Gasser S, Martino J, Martinoni M, Matsuda R, Duffau H: Selection of intraoperative tasks for awake mapping based on relationships between tumor location and functional networks. J Neurosurg 119:1380 1394, 2013

16. Goodglass H, Kaplan E: The Assessment of Aphasia and Related Disorders. Philadelphia: Lea \& Febiger, 1983

17. Graf Estes K, Evans JL, Else-Quest NM: Differences in the nonword repetition performance of children with and without specific language impairment: a meta-analysis. J Speech Lang Hear Res 50:177-195, 2007

18. Halwani GF, Loui P, Rüber T, Schlaug G: Effects of practice and experience on the arcuate fasciculus: comparing singers, instrumentalists, and non-musicians. Front Psychol 2:156, 2011

19. Havas V, Gabarrós A, Juncadella M, Rifa-Ros X, Plans G, Acebes JJ, et al: Electrical stimulation mapping of nouns and verbs in Broca's area. Brain Lang 145-146:53-63, 2015

20. Hickok G, Poeppel D: The cortical organization of speech processing. Nat Rev Neurosci 8:393-402, 2007

21. Ibertsson T, Willstedt-Svensson U, Radeborg K, Sahlén B: A methodological contribution to the assessment of nonword repetition-a comparison between children with specific language impairment and hearing-impaired children with hearing aids or cochlear implants. Logoped Phoniatr Vocol 33:168-178, 2008

22. Jefferies E, Jones RW, Bateman D, Ralph MAL: A semantic contribution to nonword recall? Evidence for intact phonological processes in semantic dementia. Cogn Neuropsychol 22:183-212, 2005

23. Lazar M, Alexander AL, Thottakara PJ, Badie B, Field AS: White matter reorganization after surgical resection of brain tumors and vascular malformations. AJNR Am J Neuroradiol 27:1258-1271, 2006

24. López-Barroso D, Catani M, Ripollés P, Dell'Acqua F, Rodríguez-Fornells A, de Diego-Balaguer R: Word learning is mediated by the left arcuate fasciculus. Proc Natl Acad Sci U S A 110:13168-13173, 2013

25. Louis DN, Ohgaki H, Wiestler OD, Cavenee WK, Burger PC, Jouvet A, et al: The 2007 WHO classification of tumours of the central nervous system. Acta Neuropathol 114:97-109, 2007

26. Marchina S, Zhu LL, Norton A, Zipse L, Wan CY, Schlaug G: Impairment of speech production predicted by lesion load of the left arcuate fasciculus. Stroke 42:2251-2256, 2011

27. McCarthy R, Warrington EK: A two-route model of speech production. Evidence from aphasia. Brain 107:463-485, 1984

28. McKean C, Letts C, Howard D: Developmental change is key to understanding primary language impairment: the case of phonotactic probability and nonword repetition. J Speech Lang Hear Res 56:1579-1594, 2013

29. Moritz-Gasser S, Duffau H: The anatomo-functional connectivity of word repetition: insights provided by awake brain tumor surgery. Front Hum Neurosci 7:405, 2013

30. Morrison MA, Tam F, Garavaglia MM, Golestanirad L, Hare
GMT, Cusimano MD, et al: A novel tablet computer platform for advanced language mapping during awake craniotomy procedures. J Neurosurg 16:1-7, 2015

31. Ober BA, Shenaut GK: Repetition priming of words and nonwords in Alzheimer's disease and normal aging. Neuropsychology 28:973-983, 2014

32. Ojemann G, Ojemann J, Lettich E, Berger M: Cortical language localization in left, dominant hemisphere. An electrical stimulation mapping investigation in 117 patients. 1989. J Neurosurg 108:411-421, 2008

33. Ojemann JG, Ojemann GA, Lettich E: Cortical stimulation mapping of language cortex by using a verb generation task: effects of learning and comparison to mapping based on object naming. J Neurosurg 97:33-38, 2002

34. Oldfield RC: The assessment and analysis of handedness: the Edinburgh inventory. Neuropsychologia 9:97-113, 1971

35. Olsen KD, Lewis JE: Carcinoma ex pleomorphic adenoma: a clinicopathologic review. Head Neck 23:705-712, 2001

36. Parker Jones O, Prejawa S, Hope TMH, Oberhuber M, Seghier ML, Leff AP, et al: Sensory-to-motor integration during auditory repetition: a combined fMRI and lesion study. Front Hum Neurosci 8:24, 2014

37. Quigg M, Fountain NB: Conduction aphasia elicited by stimulation of the left posterior superior temporal gyrus. $\mathbf{J}$ Neurol Neurosurg Psychiatry 66:393-396, 1999

38. Quigg M, Geldmacher DS, Elias WJ: Conduction aphasia as a function of the dominant posterior perisylvian cortex. Report of two cases. J Neurosurg 104:845-848, 2006

39. Rauschecker AM, Deutsch GK, Ben-Shachar M, Schwartzman A, Perry LM, Dougherty RF: Reading impairment in a patient with missing arcuate fasciculus. Neuropsychologia 47:180-194, 2009

40. Rauschecker JP: Ventral and dorsal streams in the evolution of speech and language. Front Evol Neurosci 4:7, 2012

41. Rofes A, Miceli G: Language mapping with verbs and sentences in awake surgery: a review. Neuropsychol Rev 24:185-199, 2014

42. Rofes A, Spena G, Miozzo A, Fontanella MM, Miceli G: Advantages and disadvantages of intraoperative language tasks in awake surgery: a three-task approach for prefrontal tumors. J Neurosurg Sci 59:337-349, 2015

43. Rorden C, Brett M: Stereotaxic display of brain lesions. Behav Neurol 12:191-200, 2000

44. Roux FE, Durand JB, Jucla M, Réhault E, Reddy M, Démonet JF: Segregation of lexical and sub-lexical reading processes in the left perisylvian cortex. PLoS One 7:e50665, 2012

45. Roux FE, Miskin K, Durand JB, Sacko O, Réhault E, Tanova $\mathrm{R}$, et al: Electrostimulation mapping of comprehension of auditory and visual words. Cortex 71:398-408, 2015

46. Saur D, Kreher BW, Schnell S, Kümmerer D, Kellmeyer P, Vry MS, et al: Ventral and dorsal pathways for language. Proc Natl Acad Sci U S A 105:18035-18040, 2008

47. Shinoura N, Midorikawa A, Onodera T, Tsukada M, Yamada $\mathrm{R}$, Tabei Y, et al: Damage to the left ventral, arcuate fasciculus and superior longitudinal fasciculus-related pathways induces deficits in object naming, phonological language function and writing, respectively. Int J Neurosci 123:494-502, 2013

48. Sierpowska J, Gabarrós A, Fernandez-Coello A, Camins À, Castañer S, Juncadella M, et al: Morphological derivation overflow as a result of disruption of the left frontal aslant white matter tract. Brain Lang 142:54-64, 2015

49. Sierpowska J, Gabarrós A, Ripollés P, Juncadella M, Castañer S, Camins Á, et al: Intraoperative electrical stimulation of language switching in two bilingual patients. Neuropsychologia 51:2882-2892, 2013

50. Snodgrass JG, Vanderwart M: A standardized set of 260 pictures: norms for name agreement, image agreement, fa- 
miliarity, and visual complexity. J Exp Psychol Hum Learn 6:174-215, 1980

51. Thiebaut de Schotten M, ffytche DH, Bizzi A, Dell'Acqua F, Allin M, Walshe M, et al: Atlasing location, asymmetry and inter-subject variability of white matter tracts in the human brain with MR diffusion tractography. Neuroimage 54:4959,2011

52. Thiebaut de Schotten M, Tomaiuolo F, Aiello M, Merola S, Silvetti M, Lecce F, et al: Damage to white matter pathways in subacute and chronic spatial neglect: a group study and 2 single-case studies with complete virtual "in vivo" tractography dissection. Cereb Cortex 24:691-706, 2014

53. Tuomiranta LM, Càmara E, Froudist Walsh S, Ripollés P, Saunavaara JP, Parkkola R, et al: Hidden word learning capacity through orthography in aphasia. Cortex 50:174-191, 2014

54. Vassal F, Schneider F, Sontheimer A, Lemaire JJ, Nuti C: Intraoperative visualisation of language fascicles by diffusion tensor imaging-based tractography in glioma surgery. Acta Neurochir (Wien) 155:437-448, 2013

55. Wang R, Benner T, Sorensen A, Wedeen VJ: Diffusion toolkit: A software package for diffusion imaging data processing and tractography. (http://trackvis.org/faq/2007_ISMRM_ diffusion_toolkit.pdf ) [Accessed March 11, 2016]

56. Wechsler D: Wechsler Adult Intelligence Scale-Third Edition (WAIS-III): Administration and Scoring Manual. San Antonio: The Psychological Corp., 1997

57. Weiller C, Bormann T, Saur D, Musso M, Rijntjes M: How the ventral pathway got lost: and what its recovery might mean. Brain Lang 118:29-39, 2011

\section{Disclosures}

The authors report no conflict of interest concerning the materials and methods used in this study or the findings specified in this paper.

\section{Author Contributions}

Conception and design: Rodriguez-Fornells, Sierpowska, Gabarrós. Acquisition of data: Sierpowska, Gabarrós, Fernandez-Coello, Camins, Castañer, Juncadella. Analysis and interpretation of data: Rodriguez-Fornells, Sierpowska, Gabarrós, Camins, Castañer, Morís. Drafting the article: Rodriguez-Fornells, Sierpowska. Critically revising the article: Rodriguez-Fornells, Gabarrós, Fernandez-Coello, Camins, Castañer, Juncadella, Morís. Reviewed submitted version of manuscript: all authors. Approved the final version of the manuscript on behalf of all authors: RodriguezFornells. Statistical analysis: Rodriguez-Fornells, Sierpowska, Morís. Administrative/technical/material support: Sierpowska. Study supervision: Rodriguez-Fornells.

\section{Supplemental Information \\ Online-Only Content}

Supplemental material is available with the online version of the article.

Supplemental Figure A. http://thejns.org/doi/suppl/10.3171/ 2016.2.JNS151592.

\section{Correspondence}

Antoni Rodriguez-Fornells, Basic Psychology Department, University of Barcelona, Campus de Ciències de la Salut de Bellvitge, c. Feixa Llarga, s/n, Pavelló de Govern, Edifici Modular, despatx 1.7, L'Hospitalet de Llobregat, Barcelona 08097, Spain. email: antoni.rodriguez@icrea.cat. 